\title{
ILCEA
}

Revue de l'Institut des langues et cultures

d'Europe, Amérique, Afrique, Asie et Australie

22 | 2015

La révolution théâtrale dans le Río de la Plata

\section{Introduction. - Les frontières en jeu}

\section{Cristina Breuil}

\section{OpenEdition}

Journals

Édition électronique

URL : http://journals.openedition.org/ilcea/3136

DOI : 10.4000/ilcea.3136

ISSN : 2101-0609

Éditeur

UGA Éditions/Université Grenoble Alpes

Édition imprimée

ISBN : 978-2-84310-295-0

ISSN : 1639-6073

Référence électronique

Cristina Breuil, «Introduction. - Les frontières en jeu », ILCEA [En ligne], 22 | 2015, mis en ligne le 01 mars 2015, consulté le 24 septembre 2020. URL : http://journals.openedition.org/ilcea/3136 ; DOI : https://doi.org/10.4000/ilcea.3136

Ce document a été généré automatiquement le 24 septembre 2020

(C) ILCEA 


\title{
Introduction. - Les frontières en jeu
}

\author{
Cristina Breuil
}

In memoriam Michel Lafon,

fondateur de l'Axe « Río de la Plata » de l'ILCEA 4, guide passionné, passionnant passeur de révolutions littéraires, et j'en passe...

$\mathrm{Au}$ fur et à mesure que nous avancions dans nos entretiens, je me suis aperçu que j'avais mené une vie de passeur de plus d'une façon : après avoir traversé moi-même les frontières, j'essayais d'en faciliter le passage à d'autres. Frontières d'abord entre pays, langues, cultures ; ensuite entre domaines d'étude dans le champ des sciences humaines.

Mais frontières aussi entre le banal et l'essentiel, le quotidien et le sublime, la vie matérielle et la vie de l'esprit. Dans les débats, j'aspire au rôle de médiateur. Le manichéisme et les rideaux de fer sont ce que j'aime le moins. Tzvetan TODORov (2002), Devoirs et délices. Une vie

de passeur, entretiens avec Catherine Portevin, Paris : Seuil.

Le Río de la Plata ne serait-il pas le creuset d'une révolution théâtrale plus ample, observable à l'échelle mondiale ? Telle était la question posée en amont de la rencontre des 9 et 10 avril 2013, organisée par l'ILCEA 4, qui se proposait d'analyser de manière transdisciplinaire l'apport singulier de cette zone géographique dans le renouvellement actuel des écritures et des pratiques théâtrales. C'est grâce à un dialogue entre acteurs 
du monde universitaire et du monde du théâtre que nous avons pu appréhender une (r)évolution théâtrale majeure, marquée par les traces et les silences d'une Histoire encore largement indicible, mais aussi par les signes d'une profonde revitalisation. Comme le souligne Jorge Dubatti, la post-dictature implique une appréhension nouvelle des frontières de tout théâtre national. L'histoire, la mémoire et ses reconstructions souvent audacieuses irriguent la production théâtrale de notre époque, dans un monde caractérisé par une déterritorialisation des moyens d'expression et de communication et par la multiplication des formes et des significations de l'exil. "El exilio antes era el castigo. Ahora la peor sanción es no estar adentro ni afuera. Estar entre paréntesis. Al borde siempre al borde. Suspendidos en un hilo angosto como equilibristas ${ }^{1}$." (Serrano, 2001) Et pourtant, à l'instar des personnages de Fronteras, de Santiago Serrano, l'équilibriste contemporain - dramaturge, comédien, metteur en scène, lecteur, spectateur ou chercheur - est avant tout un être libre.

Bien que nous soyons désormais dans une société de l'image et du spectaculaire, où le théâtre tend à se disséminer, dans toutes les sphères socio-politiques, dans un vaste courant de trans-théâtralisation (Dubatti), ce genre littéraire ancré dans une tradition et une pratique pluriséculaires est aussi le lieu d'où émergent tous les renouvellements possibles en termes de poétiques et de pratiques. C'est ce que révèlent chacune à leur manière et toutes ensemble les analyses réunies dans ce volume. À Buenos Aires comme à Montevideo, pour ne citer que les capitales, on observe des phénomènes locaux mais révélateurs d'un mouvement de plus grande amplitude. La multiplication des espaces atypiques de représentation théâtrale incluant différents lieux publics et privés de la ville est exposée par Nicasio Perera San Martín - à travers le cas de l'Uruguay - et par Carla Pessolano - par l'exemple de la Organización Negra des années 1984-1986 à Buenos Aires. Ces exemples illustrent bien une réalité et une évolution que les réflexions et les chiffres récents fournis par Jorge Dubatti viennent étayer de manière éloquente : un millier de représentations théâtrales en 2012 à Buenos Aires, sans compter celles, très nombreuses, qui ont eu lieu hors des espaces habituels (rues, espaces communautaires ou privés).

Laboratoire de la théâtralité (Dubatti), le Río de la Plata est un lieu où la pratique oblige sans cesse à repenser la théorie, et surtout à la décloisonner. Le surgissement du mot « teatrista » révèle d'ailleurs la diversification des rôles des différents acteurs au sein du monde du théâtre, et leur appartenance à un univers plus ample, qu'ils soient dramaturges, metteurs en scène, comédiens ou critiques... Faire du théâtre, c'est jouer avec les règles du jeu, expérimenter sans relâche et remettre en question toutes les frontières: celles des genres, des espaces, des langages et des corps. Lionel Souquet analyse les transgressions dramaturgiques à l'œuvre dans les pièces de deux écrivains argentins de l'exil, Manuel Puig et Copi, dont les personnages, évoluant aux frontières de la folie, sondent les ambiguïtés de l'identité dans des jeux de rôles qui dévoilent leurs ressorts (méta-)théâtraux, "entre métaphysique et politique ». Karen Cervera présente la singularité d'une textualité dramatique née de la collaboration entre deux écrivains argentins contemporains, Daniel Guebel et Sergio Bizzio, qui jouent à brouiller les frontières génériques et auctoriales, mais aussi celles la représentabilité. Le caractère expérimental du théâtre est également analysé par Carla Pessolano, à travers la subjectivité poétique et ses "incarnations", dans les années de postdictature, par les membres de la Organización Negra, sous la forme de «modèles vivants » transgresseurs et recréateurs de l'espace urbain. 
4 Le théâtre est devenu une véritable mise en action de l'écriture, mais aussi de plus en plus souvent une succession d'événements préalables à toute écriture, une action poétique résolument éthique et fondamentalement plurielle. À travers son parcours de dramaturge, metteur en scène et comédien, Santiago Serrano décrit comment l'expérience artistique est aussi pour lui une expérience de vie et une expérience vitale qui guide ses pas d'« acteur qui écrit » au-devant de toutes les frontières - temporelles, géographiques, linguistiques, mais aussi corporelles et existentielles -, pour l'amener à écrire depuis cette nouvelle frontière qu'il investit pleinement et d'où ses textes voyagent, souvent avec lui.

5 Le texte doit être transgressé, mis à l'épreuve, comme le montrent Karen Cervera et Santiago Serrano, tandis que les univers théâtraux se multiplient, engendrant tout autant de pratiques et de poétiques. La notion même de texte théâtral nécessite d'être repensée à l'aune de cette expérience qui en fait avant tout un « événement théâtral ». La réflexion de Marcelino Cotilla Vaca s'appuie sur cette unicité du moment de la représentation pour analyser la réception d'une œuvre de l'Argentin Claudio Tolcachir jouée en Italie, ce qui lui permet, tout en mettant en lumière des questions de compréhension interculturelle, de montrer à son tour comment le texte se transforme au fil de ses interprétations par des spectateurs de différents pays réunis dans un théâtre. Brigitte Natanson se penche également sur l'événement théâtral en analysant les bribes paratextuelles telles que les didascalies et leur pouvoir sur le texte même, montrant la singularité d'un teatro perdido œuvrant du texte à la scène. Car si le texte théâtral est caractérisé par sa vocation scénique, les modalités mêmes de son écriture sont elles aussi multiformes. De plus en plus souvent, la pièce surgit sur la scène même, dans un processus de création collective appelé couramment "écriture scénique ", (re)mettant en question et en lumière la notion même d'auctorialité, comme l'évoquent notamment les expériences de Santiago Serrano et Marcelino Cotilla Vaca.

6 Les questions qu'aborde Nicasio Perera San Martín à propos de la multiplication des lieux du théâtre au fil de l'histoire, tout en se demandant notamment si ce phénomène nous permet de continuer à parler de trames et d'œuvres théâtrales, nous invitent à une réflexion très pertinente : comment en effet ne pas perdre, d'une certaine manière, les précieux ingrédients humains et spatio-temporels qui font la magie du rituel théâtral, à force de disséminations de la théâtralité dans l'espace et le temps urbains? Cette question mérite une analyse approfondie, mais peut-être pourrait-on déjà voir, au cœur de cette pluralité théâtrale parfois confuse, les signes d'un retour à une certaine «ritualisation» du spectacle, par une quête finalement permanente de reterritorialisation qui anime les teatristas d'aujourd'hui ; mais aussi par les nouvelles formes d'historicisation que la théatralité oppose aux silences et aux simulacres de l'Histoire passée et présente.

7 Des éléments de réponse sont apportés ici même par les différentes contributions, qui montrent le surgissement de nouvelles façons de vivre et d'habiter le théâtre, mais aussi par les récits de l'expérience théâtrale pédagogique et scientifique vécue à l'université par deux étudiantes de l'Atelier théâtre espagnol'2, qui expriment bien la force de réenchantement du monde qui est à la source et à l'horizon de toute pratique théâtrale. Pour rendre compte de la participation créative, engagée et enjouée des étudiants de cet Atelier, il était en effet important de joindre à nos réflexions les deux textes écrits par Juliette Macé-Roussel et Clémentine Janin. Leur témoignage est d'autant plus précieux qu'elles racontent de façon imaginative cette aventure qu'elles 
ont partagée avec leurs camarades, guidées par Karen Cervera et moi-même, jusqu'aux répétitions finales avec Santiago Serrano et au spectacle clôturant la première journée de notre colloque. Après dix années en tant que responsable de l'Atelier de pratique théâtrale espagnol à l'université Stendhal, j'avoue que c'est un bonheur de pouvoir enfin donner la parole à des étudiants de Licence au sein d'une publication universitaire: une parole grâce à laquelle nos actions pédagogiques, scientifiques et artistiques se trouvent reliées à la source la plus vive qui puisse les animer.

Le théâtre est une caisse de résonance des arts et du monde, où dialoguent dramaturges, comédiens, spectateurs, critiques, chercheurs, sans oublier ces véritables chorégraphes audiovisuels que sont les techniciens et régisseurs qui œuvrent dans l'ombre pour donner corps à chaque présence et rendre l'événement théâtral unique ${ }^{3}$. Les textes se font et se défont, l'Histoire et les histoires se racontent et s'entrecroisent, repeuplant de sens les discours, les langages, les corps, les voix et les silences. Et c'est en jouant - en dansant -, tels des équilibristes, au cœur des multiples frontières dont nous jalonnons trop volontiers le monde, en acceptant d'oser les remettre en jeu et en question, que la théâtralité devient un espace de liberté, tandis que la notion même de frontière s'emplit de dimensions nouvelles où elle devient tour à tour tremplin, horizon, ou simplement espace (enfin) habitable. Bien que nous œuvrions désormais dans un monde si automatiquement mis en spectacle, il semblerait qu'en tant que teatristas, nous soyons plus que jamais portés par un refus de la spectacularisation systématique du monde, et par un désir de réinvention vivante de l'espace de (re)création qu'est le théâtre.

9 Le foisonnement des univers du théatre et leurs crises mêmes sont finalement une occasion d'ouvrir nos esprits et nos pratiques en renonçant à toute velléité d'« expertise » dans ce domaine, de repenser nos outils d'analyse dramaturgiques, et même de les forger. Le théâtre «teatra ${ }^{4}$ » pour reprendre ce verbe néologique rappelé par Jorge Dubatti en clôture de notre colloque, exprimant ainsi la singularité et la créativité de l'événement que constitue le théâtre, mais aussi tout colloque sur le théâtre. Expériences vécues, espaces multiples, viennent revitaliser une réflexion critique qui, à l'image de ces deux mémorables journées d'avril 2013 en compagnie d'invités venus de différents pays d'Europe et du Río de la Plata ${ }^{5}$, nous a menés à vivre le théâtre comme frontière qui réunit, individuellement et collectivement, et que nous pouvons habiter ensemble.

10 Ce n'est d'ailleurs pas seulement dans le domaine du théâtre que l'on peut parler d'une véritable révolution dans le Río de la Plata : la réflexion finale d'Émilie Delafosse sur les blogs d'écrivains argentins, menée parallèlement à notre travail sur le théâtre - et en dialogue avec lui au cours de nos séminaires de l'Axe Río de la Plata du CERHIUS -, fait écho aux questionnements des teatristas, car elle montre une autre forme de mise en scène des œuvres littéraires et de leurs auteurs. Phénomène mondial, ces nouveaux espaces virtuels de création à la fois personnels et collectifs constituent eux aussi une révolution littéraire, dans la mesure où ils permettent de faire circuler les textes - souvent accompagnés d'images et parfois de sons - et de suivre leurs évolutions, dévoilant ainsi les états mouvants d'une œuvre singulière. Les blogs font jouer, à leur manière, «les frontières du texte, de l'œuvre, et des genres ", comme l'écrit Émilie Delafosse. Ainsi font les textes dramatiques, dont la vocation scénique les rend adaptables à l'infini. Face aux traditionnelles difficultés d'édition du théâtre, les 
dramaturges ont d'ailleurs largement recours à ce mode interactif de diffusion de leur œuvre et de ses surprenantes métamorphoses.

11 Nécessairement réduites, les frontières géographiques et génériques assignées au propos du colloque de 2013 étaient un jalon provisoire et fécond, la première étape d'un dialogue international que nous espérons voir se poursuivre dans les prochaines années, entre orillas, dans le même esprit d'ouverture transdisciplinaire qui a animé cette première rencontre passionnante de femmes et d'hommes épris de théatre - et pris au jeu de la créativité.

\section{NOTES}

1. Ces mots du dramaturge argentin Santiago Serrano ouvrent l'épigraphe de sa pièce Fronteras, dont des fragments ont constitué le fil conducteur du spectacle Orillas joué, à l'occasion de cette rencontre des 9 et 10 avril 2013, par les étudiants de l'Atelier théâtre espagnol Lansad. Fronteras avait été jouée par des étudiants de l'Atelier en 2006, à l'occasion d'une première résidence à Grenoble du dramaturge, et a été étudiée dans le cadre d'un cours de littérature contemporaine en Licence 1 LLCE à l'université Stendhal en 2013-2014. La pièce a connu des adaptations au fil de ses représentations dans différents pays, et notamment une réécriture par l'auteur en 2014.

2. L'Atelier théâtre espagnol Lansad de l'université Stendhal a associé à cette manifestation scientifique une réflexion et une mise en pratique pédagogiques et artistiques avec le spectacle théâtral Orillas, un montage inédit de fragments de textes de sept dramaturges contemporains du Río de la Plata: Santiago Serrano, Mariana Eva Pérez, Rafael Spregelburd, Daniel Guebel (Argentine), Carlos Liscano, Beatriz Corbella, Renzo Dodera (Uruguay). Deux de ces dramaturges étaient présents à la manifestation: Santiago Serrano et Mariana Eva Pérez. Cette création est une exploration des dynamiques de la frontière et de l'identité. Le programme détaillé de ce spectacle apparaît dans cet ouvrage, accompagnant les témoignages des étudiantes. Il aurait d'ailleurs été intéressant de pouvoir intégrer d'autres témoignages de cette aventure, mais cela fera l'objet de prochaines publications sur la pratique théâtrale à l'université.

3. Je ne remercierai jamais assez Cécile Chemin, régisseuse lumière et son à l'Amphidice de l'université Stendhal, pour ces onze années de travail passionnant en sa compagnie, pour sa pédagogie, pour son regard toujours pertinent sur nos spectacles, qu'elle a transformés en une multitude de tableaux vivants inoubliables (sans parler l'espagnol pourtant!). Les archives audiovisuelles de l'Atelier théâtre en gardent la mémoire, rassemblant les films des spectacles de ces dix dernières années réalisés par Cédric Augot du Service audiovisuel, et des photos réalisées par Éric Chamberod (certaines sont consultables sur le site <http://theatrespagnol.free.fr>).

4. Le théâtre "théâtre »... On retrouve ce néologisme notamment dans les recherches de Jorge Dubatti (2009), El teatro teatra. Nuevas orientaciones en teatrología, Bahía Blanca: Ediciones de la Universidad Nacional del Sur.

5. On ne peut qu'espérer que les représentants de l'Uruguay soient plus nombreux pour une prochaine édition! Et bien sûr que cette réflexion se prolonge dans les prochaines années à l'échelle de toute l'Amérique latine. 


\section{AUTEUR}

CRISTINA BREUIL

Université Grenoble Alpes, ILCEA 4-CERHIUS 